\title{
Calcified miliary brain metastases with mitochondrial inclusion bodies
}

\author{
Tsuneo Yamazaki, Yasuo Harigaya, Osamu Noguchi, Koichi Okamoto, Shunsaku Hirai
}

\begin{abstract}
A patient with calcified miliary brain metastases from lung adenocarcinoma is reported. Electron microscopic study of the metastatic tumour cells showed membranous inclusion bodies in mitochondria.
\end{abstract}

(F Neurol Neurosurg Psychiatry 1993;56:110-111)

Although metastasis to the CNS occur frequently in patients with systemic cancer ${ }^{1}$ (in the majority brain lesions are multiple ${ }^{2}$ ), only about $5 \%$ of those with multiple metastases have more than five metastatic lesions. ${ }^{2}$ In addition, calcification of metastatic brain tumours is rare. ${ }^{3}$ This report describes a case of numerous calcified brain metastases from lung cancer showing unusual mitochondrial changes.

\section{Case report}

A previously healthy, 58 year old Japanese man developed progressive dementia and mental depression over a 3 month period. On admission, he showed a very slight left hemiparesis with meningeal irritation but without papilledoema. There was hyperreflexia on the left side, but the plantar responses were both flexor. He had a right gaze preference and left homonymous hemianopia.

EEG recorded a diffuse slowing in both hemispheres. Lumbar puncture revealed high opening pressure $\left(230 \mathrm{mmH}_{2} \mathrm{O}\right)$ and elevated white cell count $\left(61 / \mathrm{mm}^{3}\right)$. The cell differential was $90 \%$ lymphocytes, $6 \%$ monocytes, and $4 \%$ neutrophils. CSF protein level was $94 \mathrm{mg} / \mathrm{dl}$, and glucose $36 \mathrm{mg} / \mathrm{dl}$. All three cytological examinations of CSF specimens obtained at two week intervals showed atypical cells which had a large nucleus but which were not established as tumour cells. Multiple CSF cultures were negative. Blood and serological examinations were normal except for a high titre of carcinoembryonic antigen $(134 \mathrm{U} / \mathrm{ml})$. Repeated examinations for systemic malignancy were negative.

Brain CT showed numerous scattered punctate, high-density nodules and multiple subcortical low density areas throughout both cerebral (fig $a$ and $b$ ) and cerebellar hemispheres. The nodules were frequently present in areas in contact with CSF but showed no mass effect. There was no enhancement of nodules after contrast medium injection. The same abnormal nodules were seen on MRI. PET scan showed decreased blood flow and brain metabolism throughout the cerebrum. Angiography of both internal carotid and vertebral arteries was normal.

An open biopsy with wedge resection of a nodule in the left parietal lobe was performed. Histology showed metastatic adenocarcinoma with calcification within the necrotic parts. Electron microscopic examination of the tumour cells demonstrated that some mitochondria contained membranous inclusion bodies (fig e and $\mathrm{f}$ ). These membranous bodies were not observed in other intracytoplasmic organelles.

After the biopsy procedure adenocarcinoma of the lung was confirmed at the ninth cytological examination of sputum. Brain CT performed four months after the patient's admission showed that the number of metastatic calcifications had increased and that each nodule had enlarged (fig $\mathrm{c}$ and $\mathrm{d}$ ). He began to have convulsions and died after 8 months in hospital. Necropsy was declined by his family.

\section{Discussion}

Madow and Alpers ${ }^{4}$ first used the histopathological term "encephalitic metastatic carcinoma" to describe numerous inconspicuous plaques formed exclusively in the grey matter. However, they did not observe nodules which were visible to the naked eye or calcification. There have been only two cases of multiple calcified brain metastases detected on brain CT and confirmed by histological examination. ${ }^{56}$ The primary lesions in these cases were lung adenocarcinoma ${ }^{5}$ and pancreatic acinarcell carcinoma. ${ }^{6}$ The observations in these cases and in our case suggest a close relationship between such a characteristic metastatic pattern and adenocarcinoma. None of the three cases showed contrast enhancement on CT imaging. Repeated brain CT showed that the size of the calcified nodules continued to increase, although they might grow slower than ordinary metastatic brain tumours.

It is known that artificial myelin figures are found in mitochondria due to inadequate glutaraldehyde fixation. ${ }^{7}$ However, the mitochondrial changes we have described were observed only in tumour cells and may represent or reflect high rate of metabolism in such malignant cells. 


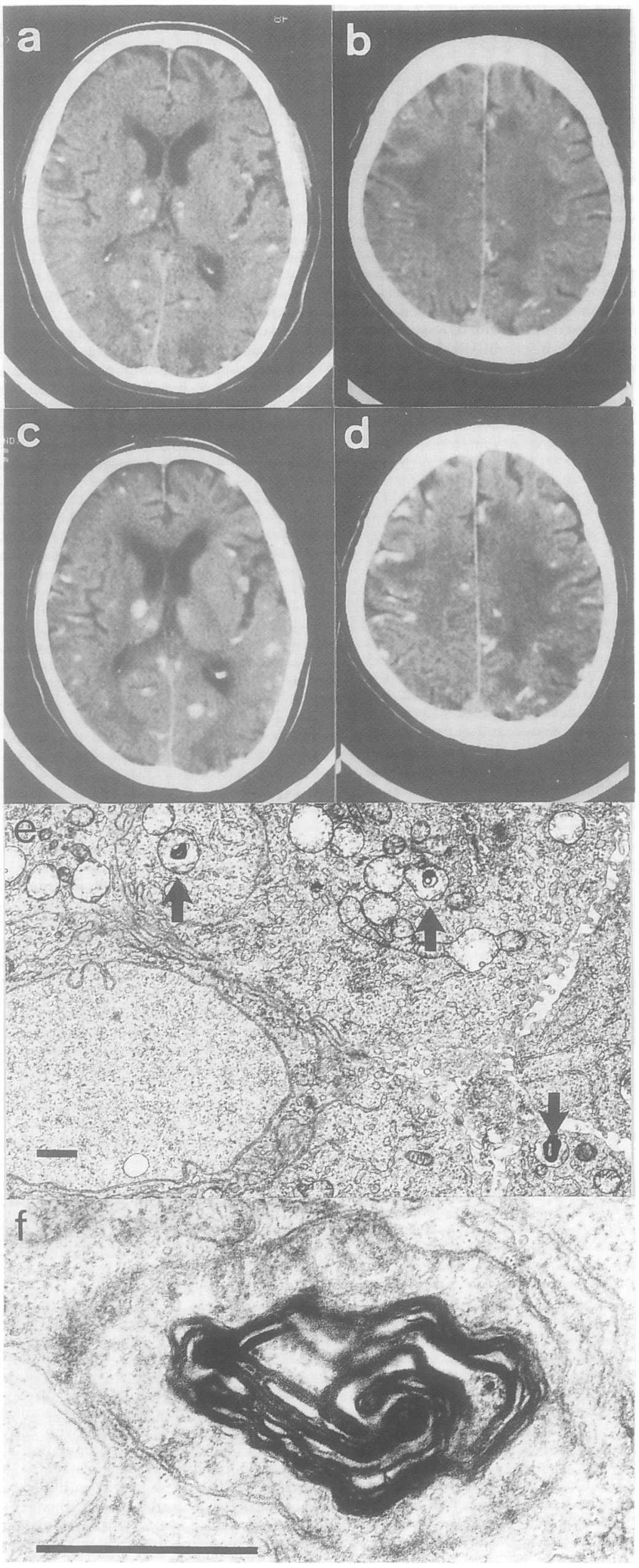

Figure 1 Non-contrast CT scans obtained at admission ( $a$ and $b$ ) and four months later ( $c$ and d) show scattered high density spots and subcortical low-density areas. An electron micrograph of tumour cells showing mitochondrial inclusion bodies ( $e$; arrows and $f)(e ; 4,800 \times$, $B a r=1 \mu \mathrm{m}$ and $\mathrm{f} ; 55,000 \times, \mathrm{Bar}=0.5 \mu \mathrm{m})$.

Posner JB, Chernick NL. Intracranial metastases from systemic cancer. Adv Neurol 1978;19:579-91.

2 Takamura K, Sano K, Hojo S, Hirano A. Metastatic tumours of the central nervous system. Tokyo:Igaku-Shoin, 1982.

3 Griffiths T. Observations on cranial radiography in a series of intracranial tumours. Br $\mathcal{F}$ Radiol 1957;30:57-69.

4 Madow L, Alpers BJ. Encephalitic form of metastatic carcinoma. Arch Neurol Psychiat 1951;65:161-73.

5 Fukuda $\mathrm{Y}$, Homma T, Kohga $\mathrm{H}$, et al. A lung cancer case with numerous calcified metastatic nodules of the brain Neuroradiology 1988;30:265-8.

6 Callizo JRA, Gimenez-Mas JA, Martin J, Lacasa J. Calcified brain metastases from acinar-cell carcinoma of pancreas. Neuroradiology 1989;31:200.

7 Ghadially FN. Ultrastructural pathology of the cell and matrix, 3rd ed. London: Butterworths, 1988. 